\title{
$B S G$ and MCT1 Genetic Variants Influence Survival in Multiple Myeloma Patients
}

\author{
Piotr Lacina ${ }^{1, *}$, Aleksandra Butrym ${ }^{2}$, Grzegorz Mazur ${ }^{2}$ and Katarzyna Bogunia-Kubik ${ }^{1}$ \\ 1 Laboratory of Clinical Immunogenetics and Pharmacogenetics, Hirszfeld Institute of Immunology and \\ Experimental Therapy, Polish Academy of Sciences, 53-114 Wrocław, Poland; bogunia@iitd.pan.wroc.pl \\ 2 Department of Internal, Occupational Diseases, Hypertension and Clinical Oncology, Wroclaw Medical \\ University, 50-556 Wrocław, Poland; aleksandra.butrym@gmail.com (A.B.); \\ grzegorz.mazur@umed.wroc.pl (G.M.) \\ * Correspondence: piotr.lacina@iitd.pan.wroc.pl; Tel.: +48-713-709-961
}

Received: 27 February 2018; Accepted: 17 April 2018; Published: 24 April 2018

\begin{abstract}
Multiple myeloma (MM) is a haematologic malignancy characterized by the presence of atypical plasma cells. Basigin (BSG, CD147) controls lactate export through the monocarboxylic acid transporter 1 (MCT1, SLC16A1) and supports MM survival and proliferation. Additionally, BSG is implicated in response to treatment with immunomodulatory drugs (thalidomide and its derivatives). We investigated the role of single nucleotide polymorphisms (SNPs) in the gene coding for BSG and SLC16A1 in MM. Following an in silico analysis, eight SNPs (four in BSG and four in SLC16A1) predicted to have a functional effect were selected and analyzed in $135 \mathrm{MM}$ patients and 135 healthy individuals. Alleles rs4919859 C, rs8637 G, and haplotype CG were associated with worse progression-free survival $(p=0.006, p=0.017, p=0.002$, respectively), while rs7556664 A, rs7169 T and rs1049434 A (all in linkage disequilibrium (LD), $r^{2}>0.98$ ) were associated with better overall survival $(p=0.021)$. Similar relationships were observed in thalidomide-treated patients. Moreover, rs4919859 C, rs8637 G, rs8259 A and the CG haplotype were more common in patients in stages II-III of the International Staging System $(p<0.05)$, while rs8259 A correlated with higher levels of $\beta$-2-microglobulin and creatinine $(p<0.05)$. Taken together, our results show that BSG and SLC16A1 variants affect survival, and may play an important role in MM.
\end{abstract}

Keywords: basigin; monocarboxylic acid transporter 1; single nucleotide polymorphisms; multiple myeloma; survival

\section{Introduction}

Multiple myeloma (MM) is the second most common human haematologic malignancy. It is characterized by presence of atypical plasma cells (myeloma cells) in the bone marrow, impaired immunoglobulin production, and presence of monoclonal protein in serum and urine [1]. The number of new MM cases is estimated at 20,000 per year in the United States, and this number is forecast to rise above 30,000 by 2032 [2].

Basigin (BSG), also known as CD147 and extracellular matrix metalloproteinase inducer (EMMPRIN), is a transmembrane glycoprotein and a member of the immunoglobulin superfamily [3]. It is widely expressed on many cells and also carries the Ok blood group in humans [4]. BSG is of major importance in myeloma cells, due to its participation in the transport of energy metabolism products, most importantly lactate anions. Lactates are produced in the glycolytic pathway and need to be removed from the cell because they decrease intracellular $\mathrm{pH}$ [5]. Lactate production is increased in cancer cells, as their reliance on the glycolytic pathway for energy production is much higher than that of normal cells-this is known as the Warburg effect. BSG is essential for lactate 
transport, although it is proteins of the monocarboxylate transporter (MCT) family that are directly responsible for it. MCTs are dependent on BSG as they are bound to it in the cell membrane, and BSG also functions as a chaperone for MCT1 and MCT4. This tight association is best seen by the fact that an MCT inhibitor was found to actually target BSG directly [3,6]. BSG overexpression was shown for many tumours, and has also been proved in multiple myeloma. Additionally, it has been demonstrated that an increase in BSG expression accompanies MM progression [7]. It was also found that MCT1 and MCT4 are overexpressed in MM, but that only MCT1 downregulation leads to decreased myeloma cell proliferation [5]. Other studies showed increased lactate export in myeloma cells, and a correlation of BSG gene expression with key regulators of Warburg effect, further confirming the role of BSG in lactate transport in MM [5,8].

A recent study showed that BSG is also implicated in MM treatment with immunomodulatory drugs (IMiDs), i.e., thalidomide and its derivatives [9]. IMiDs are known to exert their effect by binding to a protein called cereblon (CRBN) [10]. CRBN seems to promote maturation and activations of BSG and MCT1 through a chaperone-like mechanism. This process is independent of ubiquitination of proteins Ikaros and Aiolos, in which CRBN is involved, and which was shown earlier to lead to myeloma cell cycle arrest. This chaperone-like mechanism is abrogated following IMiD therapy, leading to accumulation of lactate in myeloma cells [9]. Additionally, another study showed that BSG mRNA expression is higher in patients responding to IMiD treatment than in non-responders [11].

These findings prompted us to analyse whether BSG and SLC16A1 (MCT1) gene polymorphism might affect risk, survival, or outcome of treatment in multiple myeloma patients. In our previous studies, we showed that single nucleotide polymorphisms (SNPs) located in genes associated with IMiD metabolism do affect response to therapy [12,13]. In the present work, following an in silico analysis using the National Institute of Environmental Health Sciences SNP Function Prediction tool, we picked SNPs located within the BSG and SLC16A1 genes that had minor allele frequency (MAF) in European populations higher than 0.15, and that were expected to have a functional effect [14]. Using the above criteria, eight SNPs were selected: BSG rs4919859-located in a potential transcription factor binding site, BSG rs8259-located in a potential microRNA binding site, BSG rs4682-located in an exonic splicing enhancer/silencer (ESE/ESS), BSG rs8637-located in an ESE/ESS and a potential microRNA binding site, SLC16A1 rs9429505-located in a potential microRNA binding site, SLC16A1 rs7556664-located in a potential transcription factor binding site, SLC16A1 rs7169_located in a potential microRNA binding site and SLC16A1 rs1049434-a missense Asp to Glu mutation.

\section{Materials and Methods}

\subsection{Patients and Controls}

The study included a group of 135 Polish MM patients and 135 healthy blood donors that served as controls. The group was also investigated in our previous study on CTNNB1 ( $\beta$-catenin) and $C R B N$ variants; detailed information is included there [12]. In brief, the group of patients consisted of 70'males and 65 females, median age on diagnosis was 61 years. Among patients, 35\% were in stage I, $34 \%$ in stage II, and 31\% in stage III of the disease, according to the International Staging System (ISS) criteria. $74.1 \%$ were administered thalidomide as part of the first-line treatment, mostly together with cyclophosphamide and dexamethasone.

\subsection{Genotyping}

DNA was extracted from samples of peripheral blood taken on EDTA using Maxwell 16 blood DNA purification kit (Promega Corp., Madison, WI, USA) or silica membranes (Qiagen, Hilden, Germany), following the recommendations of the manufacturers. BSG and SLC16A1 polymorphic variants were determined using the Taqman (Thermo Fisher, Waltham, MA, USA) and LightSNiP (TIB MOLBIOL, Berlin, Germany) assays. PCR was performed on a LightCycler 480 II device (Roche Diagnostics, Rotkreuz, Switzerland), according to the manufacturer's recommendations. 


\subsection{Statistical Analysis}

Linkage disequilibrium and Hardy-Weinberg equilibrium analyses were performed with the Haploview 4.2 software [15]. The null hypothesis that there is no difference between allele and genotype frequencies between patients and controls was tested with the Fisher's exact test [16]. Survival was assessed using the Kaplan-Meier method, and associations with clinical parameters were calculated using the $U$ Mann-Whitney test. Both of these analyses were performed with the real statistics resource pack for Microsoft excel 2013 (version 15.0.5023.1000, Microsoft, Redmont, Washington, DC, USA). $p<0.05$ were considered statistically significant, and those between 0.05 and 0.10 as indicative of a trend. Genotypes were tested for deviations from Hardy-Weinberg equilibrium using the $\chi^{2}$ test.

\section{Results}

\subsection{BSG and SLC16A1 Allele and Genotype Frequencies}

Patients and healthy donors were genotyped for all eight SNPs, and allele and genotype frequencies were calculated for both MM patients and controls. Frequencies for all the SNPs were in accordance with Hardy-Weinberg equilibrium in both groups. Linkage disequilibrium (LD) analysis revealed that all the BSG SNPs were in low-to-medium $\operatorname{LD}\left(r^{2}\right.$ range between 0.15 and 0.57 , see Figure 1$)$, while three of the four SLC16A1 SNPs (rs7169, rs1049434, rs7556664) were in perfect or near perfect LD $\left(r^{2} \geq 0.98\right.$, see Figure 1). Because of this, rs7556664 was used as a tag SNP for rs7169 and rs1049434, and no further analyses were performed for the two latter SNPs.

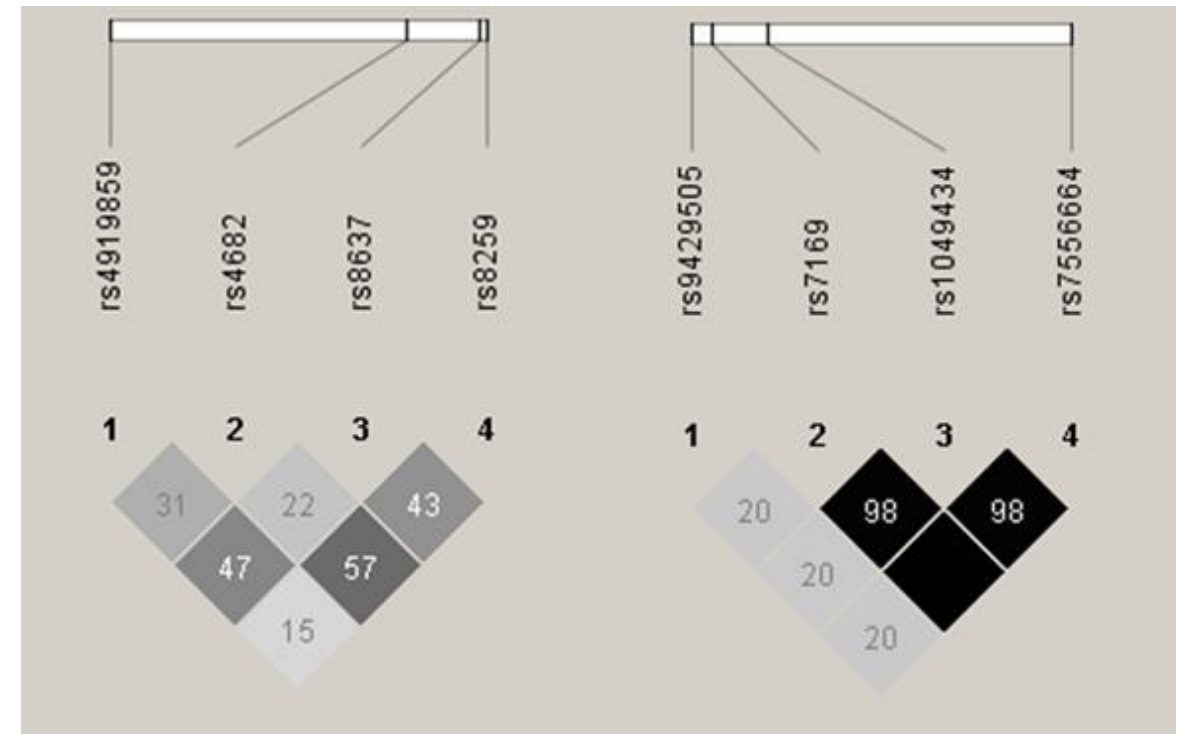

Figure 1. Linkage disequilibrium (LD) between single nucleotide polymorphisms (SNPs) under study. Basigin (BSG) SNPs are shown to the left, and monocarboxylic acid transporter 1 (SLC16A1, MCT1) to the right. Darker colour shows higher $r^{2}$ values, while the value shown inside the squares is $r^{2} \times 10^{2}$. Results as presented by the Haploview 4.2 software [15].

We did not observe any statistically significant difference in allele distribution between MM patients and healthy controls in any of the SNPs tested. Distribution of genotypes in patients and controls is presented in Table 1. 
Table 1. Distribution of BSG and SLC16A1 genotypes in multiple myeloma (MM) patients (entire patient cohort and thalidomide-treated patients only) and healthy controls.

\begin{tabular}{|c|c|c|c|c|c|c|c|}
\hline & MM Patients (All) & MM Patients (Thalidomide-Treated) & Controls & & MM Patients & MM Patients (Thalidomide-Treated) & Controls \\
\hline $\begin{array}{c}B S G \\
\text { rs } 4919859\end{array}$ & & & & $\begin{array}{l}\text { SLC16A1 } \\
\text { rs } 9429505\end{array}$ & & & \\
\hline $\mathrm{CC}$ & $15(11.2 \%)$ & $13(13.1 \%)$ & $19(14.1 \%)$ & AA & $77(57.0 \%)$ & $59(59.0 \%)$ & $67(49.6 \%)$ \\
\hline GG & $54(40.3 \%)$ & $39(39.4 \%)$ & $62(45.9 \%)$ & GG & $7(5.2 \%)$ & $7(7.0 \%)$ & $8(5.9 \%)$ \\
\hline $\begin{array}{c}B S G \\
\text { rs } 4682 \\
\end{array}$ & & & & $\begin{array}{c}S L C 16 A 1 \\
\text { rs7169 }\end{array}$ & & & \\
\hline $\mathrm{CC}$ & $3(2.2 \%)$ & $3(3.0 \%)$ & $4(3.0 \%)$ & $\mathrm{CC}$ & $22(16.3 \%)$ & $15(15.0 \%)$ & $23(17.0 \%)$ \\
\hline $\mathrm{CT}$ & $45(33.6 \%)$ & $35(35.4 \%)$ & $34(25.4 \%)$ & $\mathrm{CT}$ & $63(46.7 \%)$ & $46(46.0 \%)$ & $58(43.0 \%)$ \\
\hline TT & $86(64.2 \%)$ & $61(61.6 \%)$ & $96(71.6 \%)$ & TT & $50(37.0 \%)$ & $39(39.0 \%)$ & $54(40.0 \%)$ \\
\hline $\begin{array}{c}B S G \\
\text { rs } 8637\end{array}$ & & & & $\begin{array}{l}\text { SLC16A1 } \\
\text { rs1049434 }\end{array}$ & & & \\
\hline $\mathrm{AA}$ & $44(32.8 \%)$ & $32(32.3 \%)$ & $47(34.8 \%)$ & AA & $51(37.8 \%)$ & $39(39.0 \%)$ & $54(40.0 \%)$ \\
\hline AG & $63(47.0 \%)$ & $45(45.5 \%)$ & $61(45.2 \%)$ & AT & $62(45.9 \%)$ & $46(46.0 \%)$ & $58(43.0 \%)$ \\
\hline GG & $27(20.2 \%)$ & $22(22.2 \%)$ & $27(20.0 \%)$ & TT & $22(16.3 \%)$ & $15(15.0 \%)$ & $23(17.0 \%)$ \\
\hline $\begin{array}{c}B S G \\
\text { rs } 8259\end{array}$ & & & & $\begin{array}{l}\text { SLC16A1 } \\
\text { rs7556664 }\end{array}$ & & & \\
\hline TT & $77(57.0 \%)$ & $57(57.0 \%)$ & $78(57.8 \%)$ & $\mathrm{AA}$ & $50(37.0 \%)$ & $39(39.0 \%)$ & $54(40.0 \%)$ \\
\hline $\mathrm{TA}$ & $49(36.3 \%)$ & $35(35.0 \%)$ & $49(36.3 \%)$ & AT & $63(46.7 \%)$ & $46(46.0 \%)$ & $58(43.0 \%)$ \\
\hline AA & $9(6.7 \%)$ & $8(8.0 \%)$ & $8(5.9 \%)$ & TT & $22(16.3 \%)$ & $15(15.0 \%)$ & $23(17.0 \%)$ \\
\hline
\end{tabular}




\subsection{Associations of BSG and SLC16A1 Polymorphism with Survival}

We performed survival analyses for the SNPs regarding both progression-free (PFS) and overall survival (OS), in the whole MM patient group, and in a subgroup of patients treated with thalidomide (first-line treatment), constituting $74.1 \%$ of all patients. The analysis showed that alleles rs $4919859 \mathrm{C}$ and rs8637 G were particularly associated with adverse PFS $(p=0.006$ and $p=0.017$, for the C and G allele, respectively) (see Figure 2). Similar relationship with rs4919859 C and rs8637 G were observed in a subgroup of patients treated with thalidomide $(p=0.042$ and $p=0.065$, for the $C$ and $G$ allele, respectively). Given the similarity of rs $4919859 \mathrm{C}$ and $\mathrm{rs} 8637 \mathrm{G}$ regarding PFS and their relatively high LD, we investigated the CG haplotype and found it to be an even better predictor of adverse PFS ( $p=0.002$ and $p=0.017$, for all patients and the thalidomide-treated subgroup respectively). Furthermore, a trend towards better PFS was observed for the BSG rs8259 T allele, but not in the thalidomide-treated subgroup $(p=0.102, p=0.203$, for all patients and the thalidomide-treated subgroup respectively, see Figure 2).
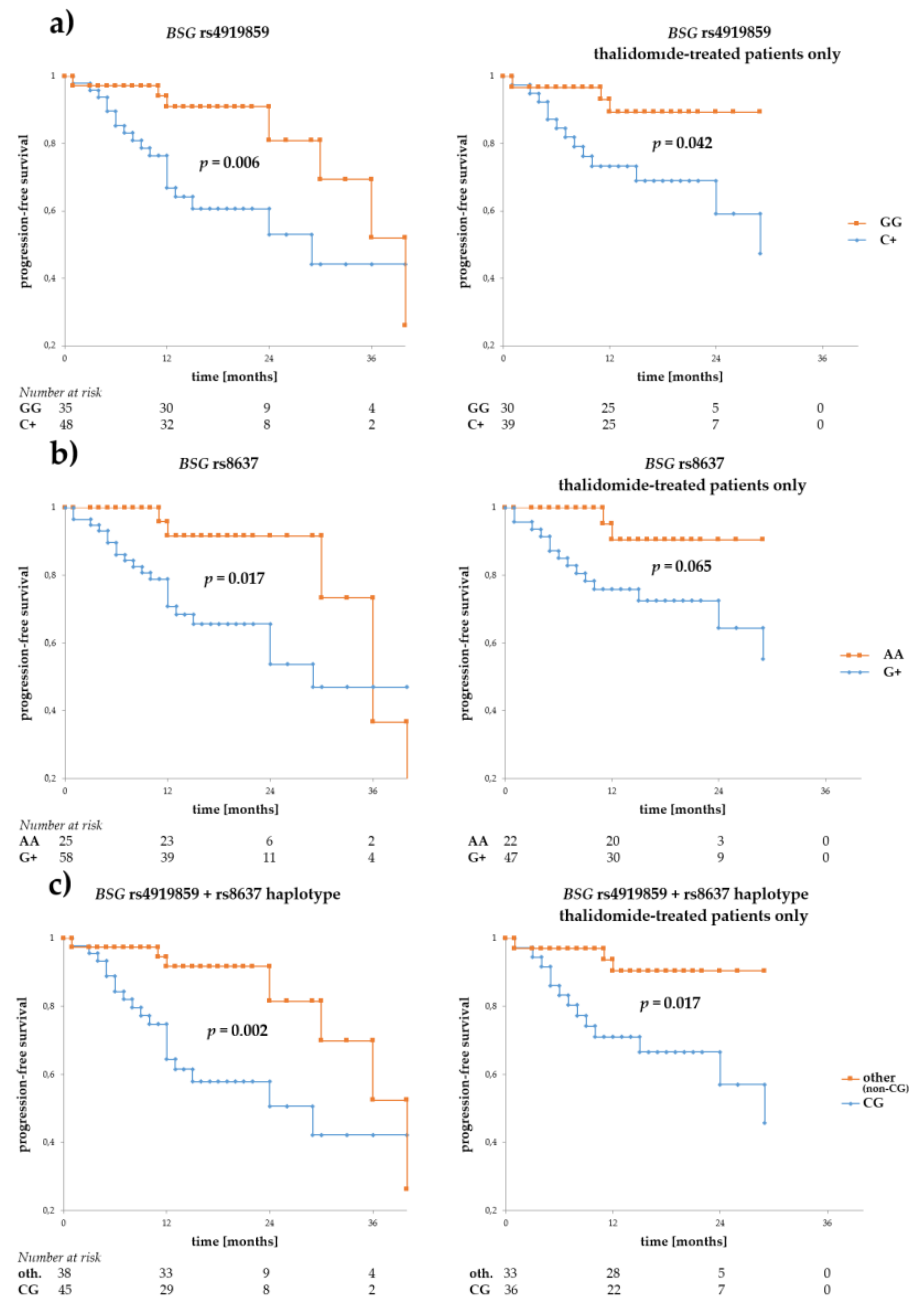

Figure 2. Progression-free survival in multiple myeloma (MM) patients with respect to BSG SNPs. Panels (a,b) show survival for rs4919859 C and rs8637 G, respectively. The two SNPs, located in a presumed transcription factor and microRNA binding sites, are in low/medium LD. Given their similar effect on progression-free (PFS), an rs4919859 C and rs8637 G (or CG, in short) haplotype was also tested for association with PFS, and is shown in panel (c). Curves to the left show PFS calculated for the entire group of patients, while those to the right show PFS calculated for the subgroup of thalidomide-treated patients. The numbers at risk show numbers of patients in the risk set (i.e., patients without disease progression, a number used to calculate survival) at a given time point on the $x$-axis. Survival was calculated using the Kaplan-Meier method and the real statistics resource pack for Microsoft excel 2013 (Microsoft). 
No statistically significant association was observed for either of the BSG rs4682 alleles. Additionally, no statistically significant associations were observed for the BSG SNPs regarding OS, although there was a trend for slightly better OS in patients with rs4682 C ( $p=0.097$ for all patients, and $p=0.090$ for the thalidomide-treated subgroup), and those with $\operatorname{rs} 8637 \mathrm{G}$ ( $p=0.092$ for all patients).

Regarding SLC16A1 SNPs, the rs7556664 A allele (and, in consequence, rs7169 T and rs1049434 A) was associated with better OS ( $p=0.021$ for all patients and $p=0.065$ for the thalidomide-treated subgroup, see Figure 3), but no such association was observed for either of the rs 9429505 alleles. However, there was a trend for better PFS in patients with rs9429505 G ( $p=0.078$, for all patients). No statistically significant association regarding PFS was observed for rs7556664.
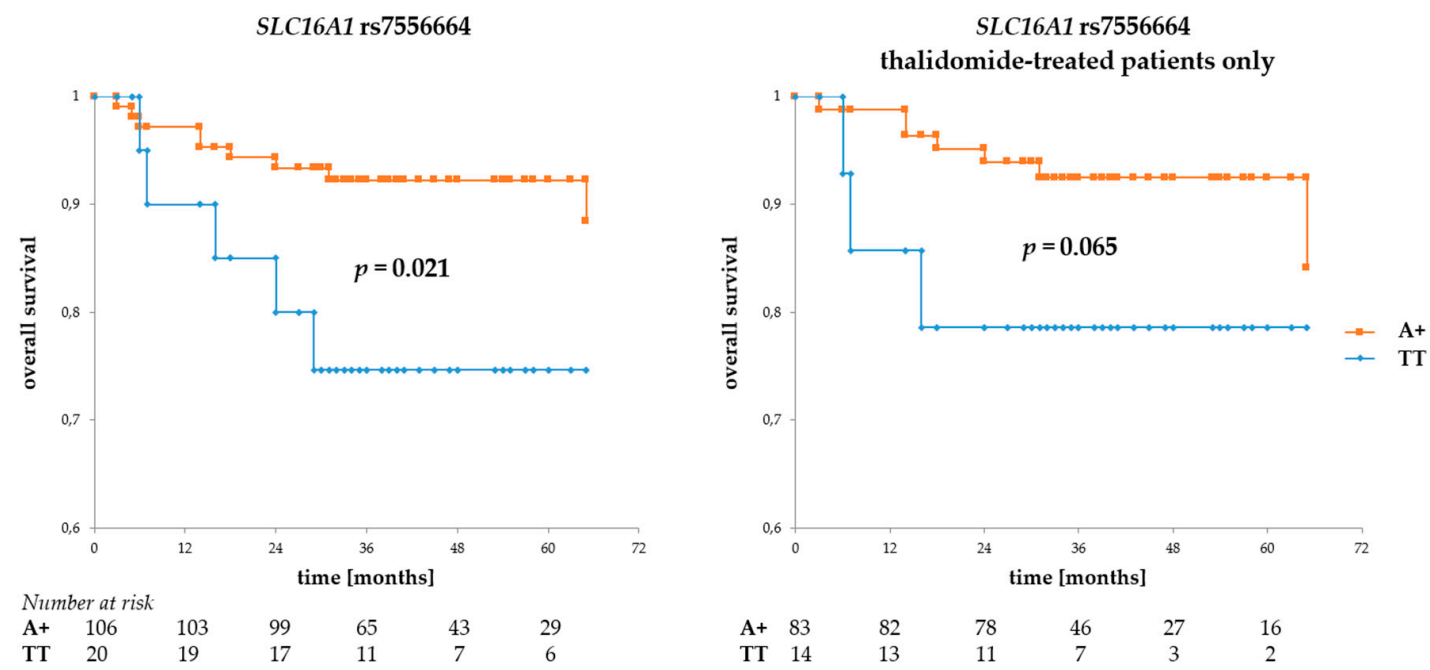

Figure 3. Overall survival in MM patients with respect to SLC16A1 rs7556664 A. This SNP is in near-perfect LD with two other SLC16A1 SNPs described in this study. Allele rs7556664 A (orange curve) also corresponds to rs1049434 A and rs7169 T; however, for the sake of simplicity, only rs7556664 $\mathrm{A}$ is shown in the figure above. The left panel shows OS calculated for the entire group of patients, while that to the right shows overall survival (OS) calculated for thalidomide-treated patients only. The numbers at risk show numbers of patients in the risk set (i.e., patients still living, a number used to calculate survival) at a given time point on the $x$-axis. Survival was calculated using the Kaplan-Meier method and the real statistics resource pack for Microsoft excel.

\subsection{Influence of BSG and SLC16A1 Polymorphism on Response to Treatment}

Keeping in mind the importance of BSG and SLC16A1 in CRBN-mediated response to IMiD therapy, we looked for associations between the SNPs under study and response to first-line treatment. Interestingly, we only observed a borderline significant association with SLC16A1 rs9429505 (the G allele was more common in patients achieving complete or very good partial remission than in patients achieving only partial remission or no remission, $p=0.048$ ), and a trend towards better response with BSG rs8259 (allele A was more common in patients achieving complete or very good partial remission, $p=0.082$ ). Both were seen in patients administered the cyclophosphamide, thalidomide, dexamethasone (CTD) regimen.

\subsection{BSG and SLC16A1 Polymorphism and Other Clinical Parameters}

We compared the occurrence of BSG and SLC16A1 variants in patients with various MM stages on diagnosis according to the international staging system (ISS). Interestingly, we found alleles rs4919859 C, rs8259 A, and rs8637 G and the CG haplotype (as defined in the Section 3.2. dealing with survival) to be more common in patients in stages II-III than in those in stage I ( $p=0.024, p=0.001, p<0.001$, $p=0.001$, respectively). A similar, although not statistically significant, relationship was also observed 
for allele rs4682 C ( $p=0.053)$. However, no association between ISS stages and SLC16A1 variants was observed.

Regarding other clinical parameters, we found rs8259 A to be associated with higher $\beta$-2-microglobulin and creatinine levels ( $p=0.022$ and $p=0.017$, respectively).

\section{Discussion}

In recent years, there have been many significant discoveries regarding the processes occurring during response to MM therapy with immunomodulatory drugs. First, CRBN was found to play a central role, and further studies showed importance of proteins Ikaros and Aiolos, as well as BSG and MCT1 $[9,10,17]$. Additionally, overexpressed BSG and MCT1 were demonstrated to be involved in energy metabolism in MM by the means of supporting the export of toxic lactate anions in myeloma cells $[5,7]$.

Our present study aimed at elucidating the role played by genetic variability in BSG and SLC16A1 (MCT1) in myeloma cells. Three SNPs located in the SLC16A1 gene turned out to be in near-perfect LD; therefore, only one of them was chosen for further analysis (rs7556664). It should, however, be noted that the observed effect of rs7556664 could also be equally attributed to either rs7169 or rs1049434.

Of the eight SNPs under study, rs4682, rs4919859, rs7169, and rs7556664 have, to the best of our knowledge, never been studied before. BSG rs8637 was mentioned in the context of carotid atherosclerotic plaques, but the study found the SNP not to be associated with risk for this disease [18]. rs8259 was described in three different non-haematologic diseases in which allele $\mathrm{T}$ was associated with lower mRNA BSG expression and lower plasma levels of soluble BSG [19-21]. This appears to agree with our findings, as rs8259 T seems to positively affect progression-free survival.

SLC16A1 rs1049434 (in near-perfect LD with rs7169 and rs7556664) was studied the most due to its exonic location, and the fact that it affects MCT1 protein structure [22-25]. It has been shown that the mutated variant (containing Glu in the protein sequence and $\mathrm{T}$ in the DNA sequence) exhibits increased lactate transport via SLA16A1, as compared to the wild type Asp/A [26]. This seems to be in line with our results showing that rs1049434 A is correlated with better survival (increased lactate transport is favourable for myeloma cells, and therefore detrimental for patient's survival).

We do not know how the other SNPs described in this study affect BSG, but given their presumed functions (as described in the introduction), we expect them to affect BSG and SLC16A1 expression (both on mRNA and protein level, depending on whether or not they are located in a potential transcription factor or miRNA binding site), or splicing (especially in the case of rs4682).

In the present study, we observed an interesting association with BSG and SLC16A1 SNPs and progression-free and overall survival. These associations show that the selected BSG and SLC16A1 alleles may influence survival in MM patients in general. However, it should be noted that some of the relationships (e.g., SLC16A1 SNPs and survival) cannot be ascertained definitively due to the relatively small size of one of the subgroups analysed. Furthermore, because many of the associations were no longer statistically significant after limiting the group to patients treated with thalidomide, we cannot decisively conclude that the various alleles affected CRBN-mediated response to thalidomide treatment. Indeed, it is possible that the observable effect might have been exclusively due to the BSG and SLC16A1 alleles variously affecting BSG-governed lactate export. As described in the results section, we found correlations with response to thalidomide treatment, although only in the case of rs9429505 was the relationship statistically significant. This relative lack of correlations between BSG SNPs and response to treatment seems to be at odds with results of Bolomsky et al., as they showed that BSG mRNA expression was significantly higher in patients responding to IMiD therapy [11]. Interestingly though, a more recent study showed no association between BSG mRNA expression and response to IMiD treatment [27]. However, it should be noted that comparison of such studies might be elusive, as the exact therapies used (e.g., IMiD chosen, other drugs used together with IMiDs, dosages etc.) vary between studies. In addition, some studies suggest that CRBN-mediated response to IMiD is controlled largely in a post-translational manner $[9,28]$. Regardless of molecular 
explanations for the survival associations, it is interesting to note that Krönke et al. also reported an association between BSG expression levels and PFS, albeit only in patients with standard (and not high) cytogenetic risk [27].

In conclusion, our study shows that selected BSG and SLC16A1 genetic variants may affect progression-free and overall survival in multiple myeloma, and correlate with various clinical parameters of MM, such as ISS stage, $\beta$-2-microglobulin and creatinine levels. These results should be further confirmed on larger cohorts of MM patients. More studies are necessary to elucidate the precise mechanism of these variants and the means by which they affect BSG/SLC16A1 levels and functions.

Author Contributions: P.Ł. and K.B.-K. conceived and designed the experiments; P.Ł. performed the experiments; P.Ł. and K.B.-K. analysed the data; A.B., G.M. and K.B.-K. contributed materials; P.Ł. and K.B.-K. wrote the paper.

Acknowledgments: The authors thank the Regional Centre of Transfusion Medicine and Blood Bank in Wroclaw for providing control samples and are grateful to Monika Chaszczewska-Markowska for her assistance.

Conflicts of Interest: The authors declare no conflict of interest.

\section{References}

1. Raab, M.S.; Podar, K.; Breitkreutz, I.; Richardson, P.G.; Anderson, K.C. Multiple myeloma. Lancet 2009, 374, 324-339. [CrossRef]

2. Rosenberg, P.S.; Barker, K.A.; Anderson, W.F. Future distribution of multiple myeloma in the United States by sex, age, and race/ethnicity. Blood 2015, 125, 410-412. [CrossRef] [PubMed]

3. Muramatsu, T. Basigin (CD147), a multifunctional transmembrane glycoprotein with various binding partners. J. Biochem. 2016, 159, 481-490. [CrossRef] [PubMed]

4. Spring, F.A.; Holmes, C.H.; Simpson, K.L.; Mawby, W.J.; Mattes, M.J.; Okubo, Y.; Parsons, S.F. The Ok ${ }^{\mathrm{a}}$ blood group antigen is a marker for the M6 leukocyte activation antigen, the human homolog of OX-47 antigen, basigin and neurothelin, an immunoglobulin superfamily molecule that is widely expressed in human cells and tissues. Eur. J. Immunol. 1997, 27, 891-897. [CrossRef]

5. Walters, D.K.; Arendt, B.K.; Jelinek, D.F. CD147 regulates the expression of MCT1 and lactate export in multiple myeloma cells. Cell Cycle 2013, 12, 3175-3183. [CrossRef] [PubMed]

6. Kirk, P.; Wilson, M.C.; Heddle, C.; Brown, M.H.; Barclay, A.N.; Halestrap, A.P. CD147 is tightly associated with lactate transporters MCT1 and MCT4 and facilitates their cell surface expression. EMBO J. 2000, 19, 3896-3904. [CrossRef] [PubMed]

7. Arendt, B.K.; Walters, D.K.; Wu, X.; Tschumper, R.C.; Huddleston, P.M.; Henderson, K.J.; Dispenzieri, A.; Jelinek, D.F. Increased expression of extracellular matrix metalloproteinase inducer (CD147) in multiple myeloma: Role in regulation of myeloma cell proliferation. Leukemia 2012, 26, 2286-2296. [CrossRef] [PubMed]

8. Panchabhai, S.; Schlam, I.; Sebastian, S.; Fonseca, R. PKM2 and other key regulators of Warburg effect positively correlate with CD147 (EMMPRIN) gene expression and predict survival in multiple myeloma. Leukemia 2017, 31, 991-994. [CrossRef] [PubMed]

9. Eichner, R.; Heider, M.; Fernández-Sáiz, V.; van Bebber, F.; Garz, A.K.; Lemeer, S.; Rudelius, M.; Targosz, B.S.; Jacobs, L.; Knorn, A.M.; et al. Immunomodulatory drugs disrupt the cereblon-CD147-MCT1 axis to exert antitumor activity and teratogenicity. Nat. Med. 2016, 22, 735-743. [CrossRef] [PubMed]

10. Ito, T.; Ando, H.; Suzuki, T.; Ogura, T.; Hotta, K.; Imamura, Y.; Yamaguchi, Y.; Handa, H. Identification of a primary target of thalidomide teratogenicity. Science 2010, 327, 1345-1350. [CrossRef] [PubMed]

11. Bolomsky, A.; Hübl, W.; Spada, S.; Müldür, E.; Schlangen, K.; Heintel, D.; Rocci, A.; Weißmann, A.; Fritz, V.; Willheim, M.; et al. IKAROS expression in distinct bone marrow cell populations as a candidate biomarker for outcome with lenalidomide-dexamethasone therapy in multiple myeloma. Am. J. Hematol. 2017, 92, 269-278. [CrossRef] [PubMed]

12. Butrym, A.; Rybka, J.; Łacina, P.; Gębura, K.; Frontkiewicz, D.; Bogunia-Kubik, K.; Mazur, G. Polymorphisms within $\beta$-catenin encoding gene affect multiple myeloma development and treatment. Leuk. Res. 2015, 39, 1462-1466. [CrossRef] [PubMed] 
13. Butrym, A.; Łacina, P.; Rybka, J.; Chaszczewska-Markowska, M.; Mazur, G.; Bogunia-Kubik, K. Cereblon and IRF4 variants affect risk and response to treatment in multiple myeloma. Arch. Immunol. Ther. Exp. 2016, 64, 151-156. [CrossRef] [PubMed]

14. Xu, Z.; Taylor, J.A. SNPinfo: Integrating GWAS and candidate gene information into functional SNP selection for genetic association studies. Nucleic Acids Res. 2009, 37, W600-W605. [CrossRef]

15. Barrett, J.C.; Fry, B.; Maller, J.; Daly, M.J. Haploview: Analysis and visualization of LD and haplotype maps. Bioinformatics 2005, 21, 263-265. [CrossRef] [PubMed]

16. VassarStats: Website for Statistical Computation. Available online: http://vassarstats.net/tab2x2.html (accessed on 23 February 2018).

17. Lu, G.; Middleton, R.E.; Sun, H.; Naniong, M.; Ott, C.J.; Mitsiades, C.S.; Wong, K.K.; Bradner, J.E.; Kaelin, W.G., Jr. The myeloma drug lenalidomide promotes the cereblon-dependent destruction of IKAROS proteins. Science 2014, 343, 305-309. [CrossRef] [PubMed]

18. Ni, T.; Chen, M.; Yang, K.; Shao, J.; Fu, Y.; Zhou, W. Association of CD147 genetic polymorphisms with carotid atherosclerotic plaques in a Han Chinese population with cerebral infarction. Thromb. Res. 2017, 156, 29-35. [CrossRef] [PubMed]

19. Wu, L.S.; Li, F.F.; Sun, L.D.; Li, D.; Su, J.; Kuang, Y.H.; Chen, G.; Chen, X.P.; Chen, X. A miRNA-492 binding-site polymorphism in BSG (basigin) confers risk to psoriasis in central south Chinese population. Hum. Genet. 2011, 130, 749-757. [CrossRef] [PubMed]

20. Yan, J.; Mao, Y.; Wang, C.; Wang, Z. Association study between an SNP in CD147 and its expression with acute coronary syndrome in a Jiangsu Chinese population. Medicine 2015, 94, e1537. [CrossRef] [PubMed]

21. Li, M.P.; Hu, X.L.; Yang, Y.L.; Zhang, Y.J.; Zhou, J.P.; Peng, L.M.; Tang, J.; Chen, X.P. Basigin rs8259 polymorphism confers decreased risk of chronic heart failure in a Chinese population. Int. J. Environ. Res. Public Health 2017, 14, 211. [CrossRef] [PubMed]

22. Cupeiro, R.; Benito, P.J.; Maffulli, N.; Calderón, F.J.; González-Lamuño, D. MCT1 genetic polymorphism influence in high intensity circuit training: A pilot study. J. Sci. Med. Sport 2010, 13, 526-530. [CrossRef] [PubMed]

23. Sawczuk, M.; Banting, L.K.; Cięszczyk, P.; Maciejewska-Karłowska, A.; Zarębska, A.; Leońska-Duniec, A.; Jastrzęski, Z.; Bishop, D.J.; Eynon, N. MCT1 A1470T: A novel polymorphism for sprint performance? J. Sci. Med. Sport 2015, 18, 114-118. [CrossRef] [PubMed]

24. Fei, F.; Guo, X.; Chen, Y.; Liu, X.; Tu, J.; Xing, J.; Chen, Z.; Ji, J.; He, X. Polymorphisms of monocarboxylate transporter genes are associated with clinical outcomes in patients with colorectal cancer. J. Cancer Res. Clin. Oncol. 2015, 141, 1095-1102. [CrossRef] [PubMed]

25. Guo, X.; Chen, C.; Liu, B.; Wu, Y.; Chen, Y.; Zhou, X.; Huang, X.; Li, X.; Yang, H.; Chen, Z.; et al. Genetic variations in monocarboxylate transporter genes as predictors of clinical outcomes in non-small cell lung cancer. Tumour Biol. 2015, 36, 3931-3939. [CrossRef] [PubMed]

26. Sasaki, S.; Futagi, Y.; Kobayashi, M.; Ogura, J.; Iseki, K. Functional characterization of 5-oxoproline transport via SLC16A1/MCT1. J. Biol. Chem. 2015, 290, 2303-2311. [CrossRef]

27. Krönke, J.; Kuchenbauer, F.; Kull, M.; Teleanu, V.; Bullinger, L.; Bunjes, D.; Greiner, A.; Kolmus, S.; Köpff, S.; Schreder, M.; et al. IKZF1 expression is a prognostic marker in newly diagnosed standard-risk multiple myeloma treated with lenalidomide and intensive chemotherapy: A study of the German myeloma study group (DSMM). Leukemia 2017, 31, 1363-1367. [CrossRef] [PubMed]

28. Gandhi, A.K.; Mendy, D.; Waldman, M.; Chen, G.; Rychak, E.; Miller, K.; Gaidarova, S.; Ren, Y.; Wang, M.; Breider, M.; et al. Measuring cereblon as a biomarker of response or resistance to lenalidomide and pomalidomide requires use of standardized reagents and understanding of gene complexity. Br. J. Haematol. 2014, 164, 233-244. [CrossRef] [PubMed]

(C) 2018 by the authors. Licensee MDPI, Basel, Switzerland. This article is an open access article distributed under the terms and conditions of the Creative Commons Attribution (CC BY) license (http:/ / creativecommons.org/licenses/by/4.0/). 